\title{
DNA damage response by single-strand breaks in terminally differentiated muscle cells and the control of muscle integrity
}

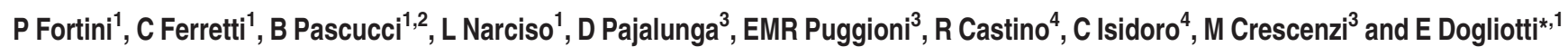

DNA single-strand breaks (SSB) formation coordinates the myogenic program, and defects in SSB repair in post-mitotic cells have been associated with human diseases. However, the DNA damage response by SSB in terminally differentiated cells has not been explored yet. Here we show that mouse post-mitotic muscle cells accumulate SSB after alkylation damage, but they are extraordinarily resistant to the killing effects of a variety of SSB-inducers. We demonstrate that, upon SSB induction, phosphorylation of $\mathrm{H} 2 \mathrm{AX}$ occurs in myotubes and is largely ataxia telangiectasia mutated (ATM)-dependent. However, the DNA damage signaling cascade downstream of ATM is defective as shown by lack of p53 increase and phosphorylation at serine 18 (human serine 15). The stabilization of p53 by nutlin-3 was ineffective in activating the cell death pathway, indicating that the resistance to SSB inducers is due to defective p53 downstream signaling. The induction of specific types of damage is required to activate the cell death program in myotubes. Besides the topoisomerase inhibitor doxorubicin known for its cardiotoxicity, we show that the mitochondria-specific inhibitor menadione is able to activate p53 and to kill effectively myotubes. Cell killing is p53-dependent as demonstrated by full protection of myotubes lacking p53, but there is a restriction of p53-activated genes. This new information may have important therapeutic implications in the prevention of muscle cell toxicity.

Cell Death and Differentiation (2012) 19, 1741-1749; doi:10.1038/cdd.2012.53; published online 15 June 2012

Cells respond to genotoxic stress by activating a signaling cascade known as the DNA damage response (DDR). The DDR is a complex interlaced network comprised of DNA damage repair factors and cell cycle regulators. ${ }^{1}$ Our knowledge of the mechanisms of DDR mainly relies on studies conducted in proliferating cells, in which the cell cycle machinery is integrated with the DNA damage signaling. Much less is known in post-mitotic cells that undergo irreversible cell cycle withdrawal. DNA repair is strongly affected by the exit from the cell cycle as revealed by downregulation of the major DNA repair pathways. ${ }^{2}$ This occurs during differentiation-associated gene reprogramming at transcriptional level as in the case of genes coding for proteins shared by DNA repair and replication (e.g., replicative DNA polymerases, Flap structure-specific endonuclease 1, proliferating cell nuclear antigen and DNA ligase 1$)^{3}$ or repair proteins that are cell-cycle related (e.g., XRCC1 (X-ray repair complementing defective repair in Chinese hamster cells 1), uracil-DNA glycosylase)., Alternatively, post-translational modifications may modify the efficiency of specific DNA repair components as in the case of transcription factor $\mathrm{II} \mathrm{H}$ that, because of reduced ubiquitination, may lead to decreased global genomic nucleotide excision repair typical of differentiated cells. ${ }^{6}$
Exposure of single-stranded (ss) DNA and/or the generation of double-strand breaks (DSB) are powerful activators of DDR by recruiting and activating two protein kinases, ataxia telangiectasia and Rad3-related (ATR) or ataxia telangiectasia mutated (ATM), respectively, at the site of DNA lesion. ${ }^{7}$ This causes the local phosphorylation of the histone $\mathrm{H} 2 \mathrm{AX}$, which is a key step in the nucleation of DDR. ${ }^{8}$ Whether singlestrand breaks (SSB) that are induced either directly or as intermediate in the base excision repair (BER) activate DDR in post-mitotic cells is still a matter of debate. SSB are the most common type of spontaneous DNA damage that arise in cells, and defects in their repair have been associated with hereditary neurodegenerative diseases. ${ }^{9}$ Although DNA damage is considered to be detrimental to cell integrity, SSB have a dual role in myotubes by coordinating physiological changes associated with the myogenic program. ${ }^{10}$ Evidence has been provided that processing of base damage by DNA glycosylases can activate the ATM-checkpoint kinase 2 (Chk2) branch in $G_{1}$ cells. ${ }^{11}$ The activation of DDR would then facilitate $\mathrm{BER}$ by phosphorylating XRCC1, the BER scaffolding protein. ATM is also activated in post-mitotic cells (human lymphocytes and rat cortical neurons) by camptothecin (CPT) that selectively traps Topoisomerase (TOP)1-linked SSB (TOP1 cleavage complexes, TOP1cc). ${ }^{12}$

\footnotetext{
${ }^{1}$ Department of Environment and Primary Prevention, Istituto Superiore di Sanità, Rome, Italy; ${ }^{2}$ Institute of Crystallography, Consiglio Nazionale delle Ricerche, Rome, Italy; ${ }^{3}$ Department of Cell Biology and Neurosciences, Istituto Superiore di Sanità, Rome, Italy and ${ }^{4}$ Department of Health Sciences, University of Piemonte Orientale, Novara, Italy

${ }^{*}$ Corresponding author: E Dogliotti, Department of Environment and Primary Prevention, Istituto Superiore di Sanità, Viale Regina Elena 299, Rome 00161, Italy. Tel: + 39 0649902580; Fax: + 39 0649903650; E-mail: eugenia.dogliotti@iss.it

Keywords: DNA damage response; DNA repair; muscle cell differentiation

Abbreviations: XRCC1, X-ray repair complementing defective repair in Chinese hamster cells 1; Apaf-1, apoptotic protease activating factor 1; ROS, radical oxygen species; MDM2, human homolog of mouse double minute 2; Bax, BCL2-associated X protein; shRNA, short hairpin RNA

Received 31.8.11; revised 12.3.12; accepted 26.3.12; Edited by RA Knight; published online 15.6.12
} 
Phosphorylation of ATM by cyclin-dependent kinase 5 seems to mediate DNA damage signaling and regulate neuronal death upon CPT treatment, ${ }^{13}$ but whether this is limited to neural tissue remains to be addressed. The complexity of DDR activation in post-mitotic cells emerges also from studies conducted in differentiated muscle cells where activation of DDR was described upon induction of DSB, but a blockade of signaling was reported downstream of ATM upon exposure to ionizing radiation (IR) but not to the TOP2 inhibitor doxorubicin (DOXO). ${ }^{14}$ Interestingly, although myotubes were resistant to $\mathrm{IR}$, they were effectively killed by DOXO.

In this study, we exploited the use of a skeletal muscle cell differentiation system to address whether and how SSB induced by different model agents activate DDR in proliferating versus post-mitotic muscle cells, and how the cross-talk between DNA repair-DNA damage signaling cell death is regulated upon irreversible exit from the cell cycle.

\section{Results}

DNA SSBs accumulate in myotubes but do not lead to cell death. In this study, we exploit the use of a skeletal muscle cell differentiation system ${ }^{15}$ to study the changes in the DDR during differentiation. In this system, actively proliferating myoblasts are induced to differentiate under appropriate cell culture conditions (Supplementary Figure 1A). The extent of differentiation after 3 days as measured by differentiation and fusion indexes, reflecting, respectively, myosin heavy chain ( $\mathrm{MHC}$ ) expression and multinucleated myotubes formation, is close to $100 \%$ (Supplementary Figure 1B). Measurements of differentiation and fusion indexes are routinely performed. The decreased
BER capacity of terminally differentiated muscle cells has been shown to lead to accumulation of DNA sSSBs upon oxidative stress. ${ }^{3}$ As shown in Figure 1a, DNA SSB also accumulate after exposure to an alkylating agent, namely methyl methanesulfonate (MMS; $1 \mathrm{mM}, 30 \mathrm{~min}$ ), which induces SSB as intermediates during the BER process. To gain insights into the fate of persistent SSB in the genome of post-mitotic muscle cells, cell survival was measured in proliferating and terminally differentiated cells following exposure to MMS and CPT by counting metabolically active cells. CPT induces TOP1-linked SSB. ${ }^{16}$ At doses that killed over $50 \%$ of the proliferating cells, myotubes were resistant to the toxic effects of DNA SSB (Figure 1b). Myotubes were also resistant to $\mathrm{H}_{2} \mathrm{O}_{2}$ (data non shown), but the comparison with myoblasts was hampered by the induction of differentiation upon $\mathrm{H}_{2} \mathrm{O}_{2}$ exposure. ${ }^{17}$ Terminally differentiated muscle cells are therefore not only resistant to IR (Figure $1 \mathrm{~b}),{ }^{14}$ but also to SSB-inducing agents.

The expression of genes involved in DDR and apoptosis is modulated during differentiation. The ability of myotubes to tolerate DNA breakage prompted us to investigate the status of DDR genes of these post-mitotic cells. Differentiation involves significant gene reprogramming, including genes involved in DDR and apoptosis. ${ }^{18}$ As shown in Figure 2a, p53 levels are high during differentiation, but strongly decline in cells undergoing terminal differentiation. Similarly, high levels of p21 seem to be required to trigger differentiation (at day 1), but then, a decrease is observed as a function of the differentiation time. It is of note that although the levels of this cyclin-dependent kinase inhibitors decrease, it is required for maintaining the post-mitotic state
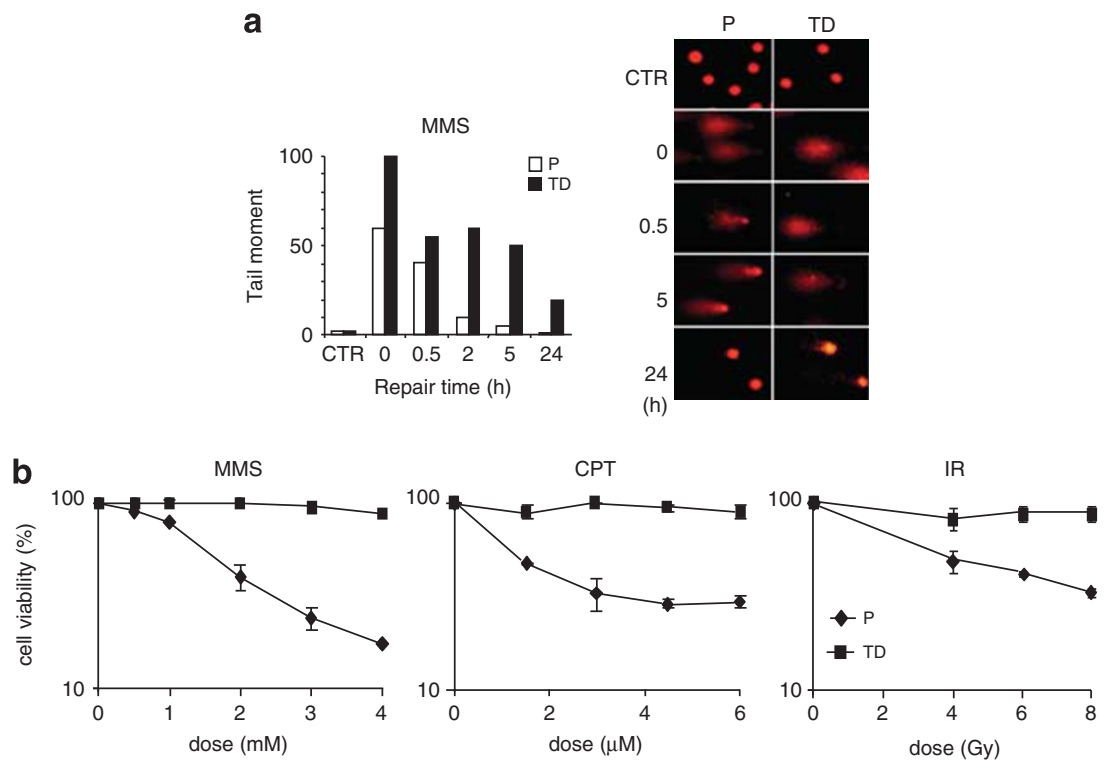

Figure 1 Terminally differentiated muscle cells accumulate DNA SSB after MMS exposure, but are resistant to their toxic effects. (a) Left: myoblasts (P) and myotubes (TD) were exposed to $1 \mathrm{mM}$ MMS for $30 \mathrm{~min}$, and DNA SSB were analyzed by the comet assay at different repair times. The average of the tail moment of at least 100 cells per experimental point is shown. Right: microphotographs of proliferating (P) and terminally differentiated (TD) cells subjected to the comet assay and stained with ethidium bromide. Representative untreated control cells (CTR) and after different post-treatment times are shown. Experiments were repeated twice and one representative experiment is shown. (b) Cell survival was measured in proliferating $(\mathrm{P})$ and terminally differentiated (TD) cells following exposure to different doses of MMS and CPT (30-min treatment and $3 \mathrm{~h}$, respectively) and IR by counting metabolically active cells as measured by CCK-8 assay $72 \mathrm{~h}$ after treatment. Error bars indicate S.D. 
of myotubes. ${ }^{19}$ The apoptosis protease-activating factor 1 (Apaf-1) is progressively downregulated in post-mitotic muscle cells. The decrease in Apaf-1 of myotubes has been associated with their resistance to apoptosis. ${ }^{20}$ As expected, myogenin, an early marker of skeletal myogenesis, increases during differentiation.

a

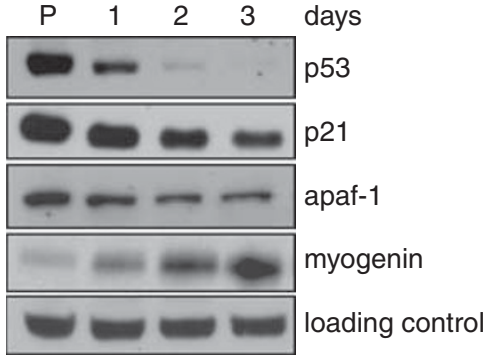

b $\begin{array}{llllllllllll}\text { CTR } & 0 & 0.5 & 2 & 5 & 24 & \text { CTR } & 0 & 0.5 & 2 & 5 & 24\end{array}$

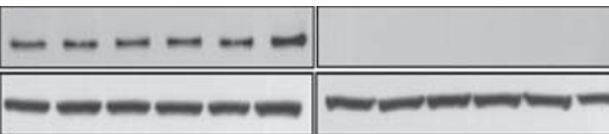

Chk1

loading control

Figure 2 The expression of DDR and apoptosis genes changes during differentiation. (a) Immunoblotting analysis was carried out by using antibodies specific for Apaf-1 $(130 \mathrm{kDa})$, p53 (53 kDa), p21 (21 kDa) and myogenin $(37 \mathrm{kDa})$ Western blot analysis was conducted on whole extracts from proliferating cells $(P)$ and at different differentiation days. Loading control: human AP endonuclease 1 , HAP1 (37 kDa). (b) Protein levels of Chk1 (56 kDa) in proliferating $(P)$ and terminally differentiated (TD) cells either untreated (control, CTR) or at different times after exposure to $\mathrm{H}_{2} \mathrm{O}_{2}(100 \mu \mathrm{M}, 30 \mathrm{~min})$. Loading control: $\beta$-tubulin $(55 \mathrm{kDa})$. The estimated MW is indicated for each protein
Downregulation and functional inactivation of genes implicated in cell cycle checkpoint signaling, such as Chk1 and ATR, is a feature of myotubes. ${ }^{21,22}$ As shown in Figure $2 b$, Chk1 is expressed in proliferating cells either untreated or exposed to hydrogen peroxide ( $100 \mu \mathrm{M} \mathrm{H}_{2} \mathrm{O}_{2}, 30 \mathrm{~min}$ ), but is missing in post-mitotic cells also after DNA damage. Conversely, ATM and Chk2 are maintained in differentiating cells also after damage. ${ }^{14,21}$ Therefore, the ATM/Chk2 module is the only kinase branch active in myotubes that present also extremely low levels of p53.

$\gamma \mathrm{H} 2 \mathrm{AX}$ response is activated in myotubes upon SSB induction and ATM is the main kinase involved. In a previous study ${ }^{3}$, we have shown that oxidative damage is able to activate the $\gamma \mathrm{H} 2 \mathrm{AX}$ response in post-mitotic muscle cells. H2AX can be phosphorylated by ATM, DNA-dependent protein kinase (DNA-PK) or ATR. In myotubes, ATR is missing but either ATM or DNA-PK could have this role. To identify the candidate kinase, myotubes were pre-treated with the ATM kinase inhibitor KU55933 for $1 \mathrm{~h}$, and the induction of phosphorylated $\mathrm{H} 2 \mathrm{AX}$ was monitored in response to $\mathrm{H}_{2} \mathrm{O}_{2}$ (100 $\mathrm{mM}, 30 \mathrm{~min}$ ) by immunofluorescence and western blotting (Figure $3 \mathrm{a}$ ). In case of terminally differentiated cells, the $\gamma \mathrm{H} 2 \mathrm{AX}$ foci were enumerated in multinucleated myotubes (Supplementary Figure S2). KU55933 fully suppressed $\mathrm{H}_{2} \mathrm{O}_{2}$-induced $\mathrm{H} 2 \mathrm{AX}$ phosphorylation, whereas NU7441 that specifically inhibits DNA-PK did not affect the $\gamma \mathrm{H} 2 \mathrm{AX}$ response (Figure $3 a$ ). Figure $3 \mathrm{~b}$ shows that ATM also participates to the activation of $\gamma \mathrm{H} 2 \mathrm{AX}$ response upon MMS exposure (3 mM, $30 \mathrm{~min}$ ), but KU55933 was effective only on $\gamma \mathrm{H} 2 \mathrm{AX}$ foci arising at later times after damage ( $24 \mathrm{~h}$ after treatment), indicating that only a subset of
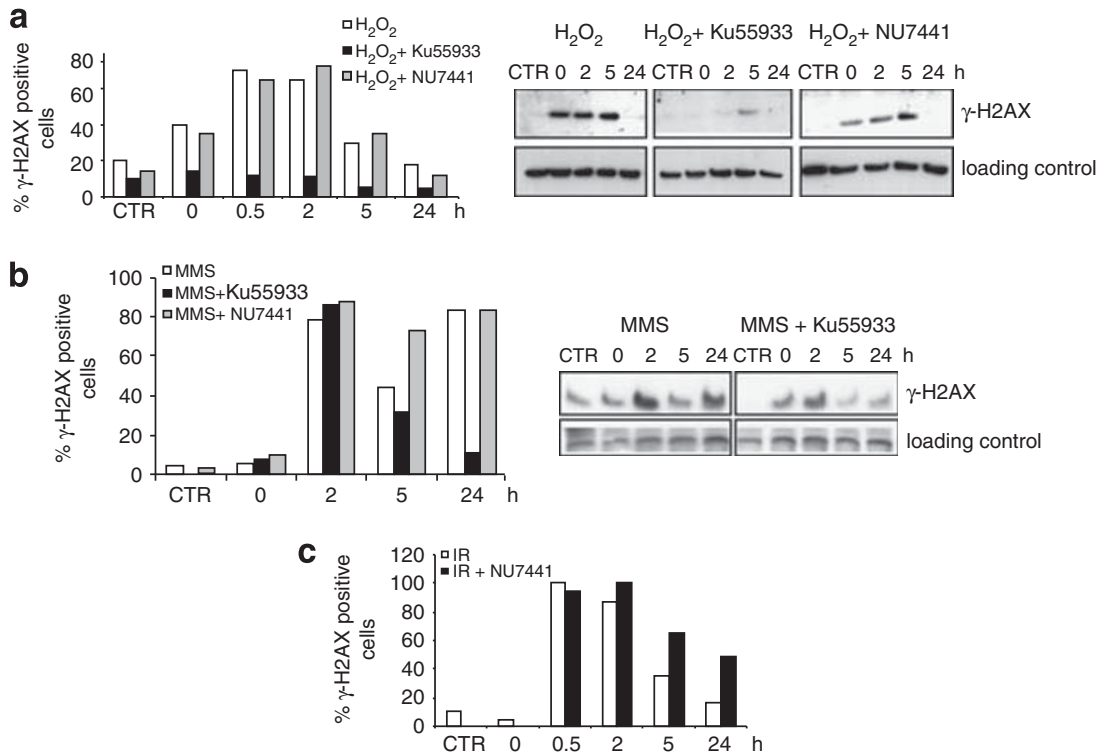

Figure $3 \gamma \mathrm{H} 2 \mathrm{AX}$ response is activated in myotubes upon SSB induction and ATM is the main kinase involved. Induction of $\gamma \mathrm{H} 2 \mathrm{AX}$ foci after $\mathrm{H}_{2} \mathrm{O}_{2}(\mathbf{a}), \mathrm{MMS}(\mathbf{b})$ or IR (c) treatment of terminally differentiated muscle cells. Myotubes were exposed to the DNA-damaging agent with or without $1 \mathrm{~h}$ pre-treatment with a specific kinase inhibitor (KU55933 or NU7441), and analyzed at different times after damage. (a) DNA-damage induction and repair as detected by $\gamma \mathrm{H} 2 \mathrm{AX}$ foci formation (left) or western blotting (right) after exposure to $\mathrm{H}_{2} \mathrm{O}_{2}(100 \mu \mathrm{M}, 30 \mathrm{~min})$. (b) DNA-damage induction and repair as detected by $\gamma \mathrm{H} 2 \mathrm{AX}$ foci formation (left) or western blotting (right) after exposure to MMS ( $3 \mathrm{mM}, 30 \mathrm{~min}$ ). (c) DNA damage induction and repair as detected by $\gamma \mathrm{H} 2 \mathrm{AX}$ foci formation after exposure to IR (2 Gy). Experiments were repeated twice and one representative experiment is shown. In the $\gamma \mathrm{H} 2 \mathrm{AX}$ foci assay, at least 200 nuclei were examined for each time point. Western blot analysis was performed on nuclear cell extracts, and the loading control is DNA polymerase $\beta$. CTR, control cells untreated 
a

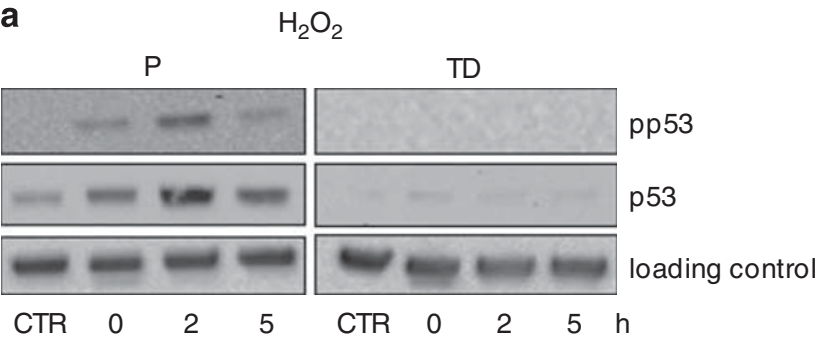

b

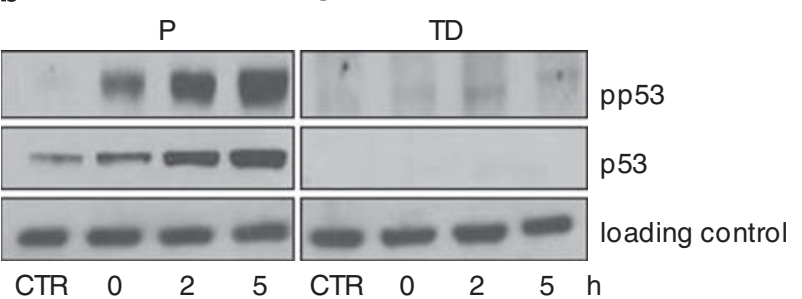

c

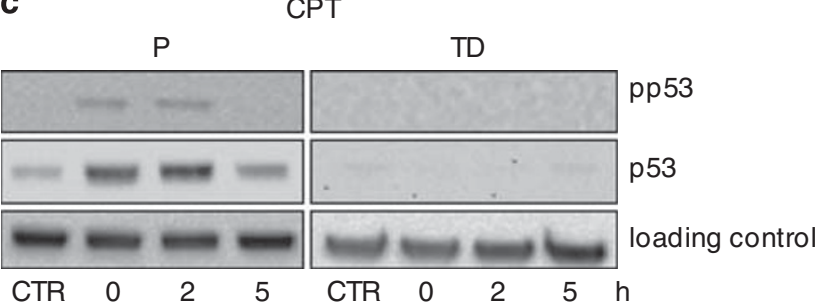

Figure 4 The p53 response is impaired in myotubes after exposure to SSB inducers. The levels of p53 and Ser 18 (human Ser 15) phosphorylated p53 (pp53) were monitored in proliferating $(\mathrm{P})$ and terminally differentiated (TD) cells at different times after exposure to (a) $\mathrm{H}_{2} \mathrm{O}_{2}(100 \mu \mathrm{M}, 30 \mathrm{~min})$, (b) MMS ( $\left.3 \mathrm{mM}, 30 \mathrm{~min}\right)$ and (c) CPT $(6 \mu \mathrm{M}, 3 \mathrm{~h})$. Western blot analysis was conducted on whole extracts. Loading control: human AP endonuclease 1, HAP1 (37 kDa). The estimated MW is indicated for each protein

alkylation-induced lesions are able to trigger ATM-dependent damage signaling. The DNA-PK inhibitor was ineffective on MMS-induced $\gamma \mathrm{H} 2 \mathrm{AX}$ response (Figure $3 \mathrm{~b}$, left).

If on one hand DNA-PK inhibition did not prevent the reversal of $\mathrm{H} 2 \mathrm{AX}$ phosphorylation upon $\mathrm{H}_{2} \mathrm{O}_{2}$ - or MMSinduced damage (Figure $3 \mathrm{a}$ ), it significantly affected H2AX dephosphorylation following exposure of myotubes to IR (Figure 3c). DNA-PK-dependent non-homologous endjoining (NHEJ) is therefore fully operational in myotubes, but pathways other than NHEJ should be involved in the repair of $\mathrm{H}_{2} \mathrm{O}_{2}$-induced lesions.

These results provide clear evidence that activation of ATM is involved in SSB signaling, but its activation is dependent on the type of damage. $\gamma \mathrm{H} 2 \mathrm{AX}$ activation initiates SSB processing via a pathway that is DNA-PK-independent.

The p53 downstream signaling is impaired in myotubes upon SSB induction. Activation of ATM is expected to lead to activation of the $p 53$ response. ${ }^{23}$ To further investigate the signaling cascade after SSB induction, the p53 gene response was investigated in proliferating and differentiated cells after exposure to SSB-inducing agents. The levels of total p53 and its serine (Ser) 18 (human Ser 15) phosphorylated form (pp53) were monitored after exposure to $\mathrm{H}_{2} \mathrm{O}_{2}$, a

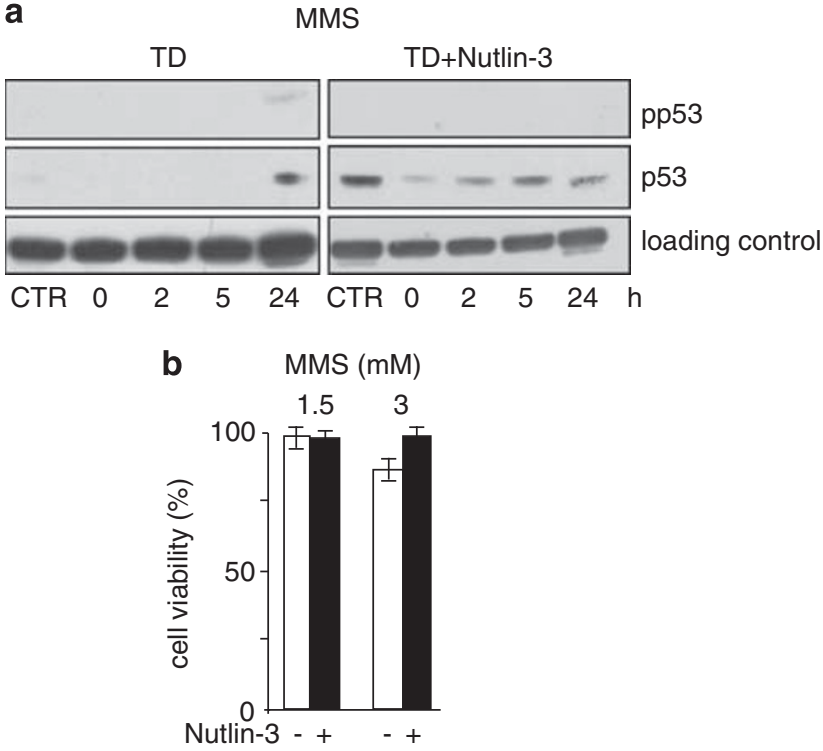

Figure 5 Nutlin 3-dependent p53 stabilization does not sensitize the myotubes to the cytotoxic effect of MMS. (a) Western blot analysis of p53 and Ser 18 (human Ser 15) phosphorylated p53 (pp53) in whole extracts of proliferating (P) and terminally differentiated (TD) cells at different times after MMS exposure $(3 \mathrm{mM}$, $30 \mathrm{~min})$. Cells were also pre-incubated with nutlin-3 $(1 \mu \mathrm{M})$ before MMS exposure. Loading control: DNA polymerase $\beta 40 \mathrm{kDa}$. The estimated MW is indicated for each protein. (b) Cell survival was measured in myotubes following exposure to 1.5 and $3 \mathrm{mM}$ MMS alone or after incubation with nutlin-3. Metabolically active cells were counted by the CCK-8 assay $48 \mathrm{~h}$ after treatment. Error bars indicate S.D. CTR, untreated control cells

MMS and CPT (Figure 4). In proliferating cells, p53 and its activated form invariably increased after SSB induction, albeit with different kinetics, but not in differentiated cells. The defective p53 response and protection from death of myotubes in response to DNA break-inducing agents might be due to the inability to stabilize p53 or to prevent transcription of some p53 target genes. To distinguish between these two possibilities, we used nutlin-3 that, by inhibiting the interaction between p53 and the human homolog of mouse double minute 2 (MDM2), stabilizes p53 and activates the p53-dependent cell death pathway. ${ }^{24}$ As shown in Figure 5a, nutlin-3 was able to stabilize p53 in untreated as well as MMS-treated myotubes; however, no toxicity was recorded (Figure 5b). The levels of p53 do not change during the additional $24 \mathrm{~h}$ that is the maximal recovery time after damage used in our experiments. As expected, nutlin-3 did not affect the phosphorylation of p53.

It is of interest to note that as a consequence of p53 stabilization, Apaf-1 mRNA levels increased (Supplementary Figure 3), but this was not sufficient to trigger cell death. ${ }^{20}$

We can conclude that upon DNA break induction, there is a blockade of the p53 downstream signaling that prevents the activation of the cell death pathway.

The p53 cell death pathway is activated in myotubes by DOXO and menadione. Although myotubes are resistant to SSB-inducing agents and IR, the p53 cell death pathway is still functional in these cells. As previously shown, ${ }^{14}$ myotubes are extremely sensitive to the 

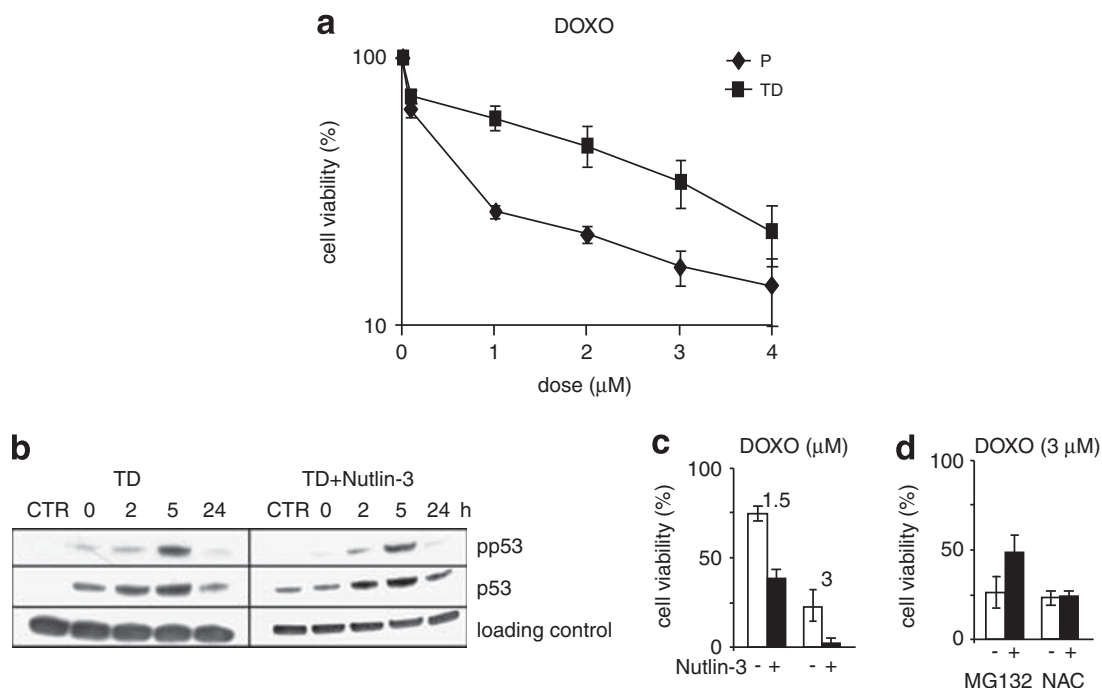

Figure 6 The p53 cell death pathway is activated in myotubes exposed to doxorubicin. (a) Cell survival was measured in proliferating (P) and terminally differentiated (TD) cells following exposure to various doses of DOXO for $3 \mathrm{~h}$. Metabolically active cells were counted by the CCK-8 assay $48 \mathrm{~h}$ after treatment. Error bars indicate S.D. (b) Western blot analysis of p53 and Ser 18 (human Ser 15) p53 (pp53) in whole extracts of myotubes at different times after DOXO exposure ( $3 \mu \mathrm{M} ; 3 \mathrm{~h}$ ) alone or preceded by incubation with nutlin-3 $(1 \mu \mathrm{M})$. CTR, untreated control cells. Loading control, $\beta$-tubulin $(55 \mathrm{kDa})$. The estimated MW is indicated for each protein. (c) Cell survival was measured in myotubes following exposure to 1.5 and $3 \mu \mathrm{M}$ DOXO with or without nutlin-3. Metabolically active cells were counted by the CCK-8 assay $48 \mathrm{~h}$ after exposure. Error bars indicate S.D. (d) Cell survival was measured in myotubes following exposure to $3 \mu \mathrm{M}$ DOXO with or without pre-incubation with MG132 (0.5 $\mu \mathrm{M}$ for $30 \mathrm{~min})$ or NAC (0.5 $\mathrm{mM}$ for $30 \mathrm{~min})$. Error bars indicate S.D.

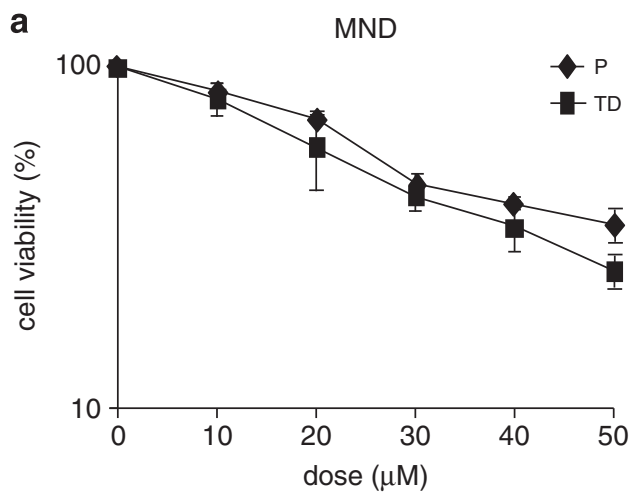

b
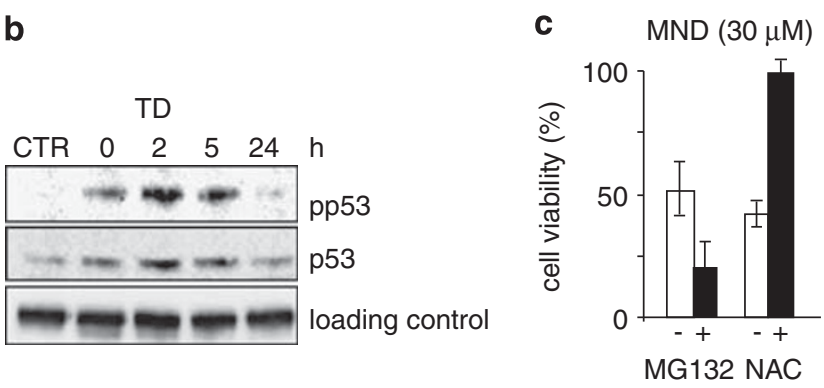

Figure 7 The 553 cell death pathway is activated in myotubes exposed to MND. (a) Cell survival was measured in proliferating $(P)$ and terminally differentiated (TD) cells following exposure to various doses of MND for $1 \mathrm{~h}$. Metabolically active cells were counted by the CCK-8 assay $48 \mathrm{~h}$ after treatment. Error bars indicate S.D. (b) Western blot analysis of p53 and Ser 18 (human Ser 15) p53 (pp53) in whole extracts of myotubes at different times after MND exposure $(20 \mu \mathrm{M}$ for $1 \mathrm{~h})$. CTR: untreated control cells. Loading control: GAPDH $(37 \mathrm{kDa})$. The estimated MW is indicated for each protein. (c) Cell survival was measured in myotubes following exposure to $30 \mu \mathrm{M}$ MND with or without pre-incubation with MG132 $(0.5 \mu \mathrm{M}$ for $30 \mathrm{~min})$ or NAC $(0.5 \mathrm{mM}$ for $30 \mathrm{~min})$. Metabolically active cells were counted by the CCK-8 assay $48 \mathrm{~h}$ after treatment. Error bars indicate S.D. anthracycline derivative DOXO (Figure 6a). Upon DOXO treatment $(3 \mu \mathrm{M}, 3 \mathrm{~h})$, the p53 response is activated in myotubes as testified by the increase of total p53 and its phosphorylated form as a function of the post-treatment time (Figure 6b). As expected, DOXO-induced cell death is reinforced $(P<0.05)$ in the presence of nutlin-3, which further stabilizes p53 (Figure 6c). DOXO induces a variety of DNA lesions, including DSB arising by TOP2 inhibition and oxidative damage, ${ }^{16}$ which might all concur to the activation of the p53-dependent cell death pathway. Several studies suggest that the toxicity exerted by TOP2 inhibitors is due to proteasomal processing of TOP2-DNA adducts that exposes TOP2-concealed DSB. ${ }^{25,26}$ In agreement with this model, the proteasome inhibitor MG132 attenuated $(P<0.05)$ DOXOinduced lethality in myotubes (Figure $6 \mathrm{~d}$ ). Conversely, the radical oxygen species (ROS)-scavenger $\mathrm{N}$-acetylcysteine (NAC) did not affect the cytotoxicity induced by DOXO (Figure 6d), indicating that in muscle cells, the redox cycling ability of DOXO does not have a major role in toxicity.

Myotubes are exquisitely sensitive to oxidative stress. The response of myotubes to the toxic effects of the mitochondria-specific inhibitor menadione (MND) ${ }^{27}$ was investigated. As shown in Figure 7a, MND induces a dramatic decrease of myotube vitality comparable to that of their proliferating counterpart. Then, p53 is also induced by MND (Figure 7b) as well as its phosphorylated form, in agreement with the idea that mitochondrial ROS rapidly activate p53. To support this mechanism of action, myotubes were fully protected $(P<0.05)$ by the cytotoxic effects of MND when the treatment was performed in the presence of the anti-oxidant NAC, whereas MG132 increased toxicity $(P<0.05$; Figure $7 \mathrm{c})$.

Together, these experiments indicate that post-mitotic muscle cells are protected by DNA breakage-induced cell 

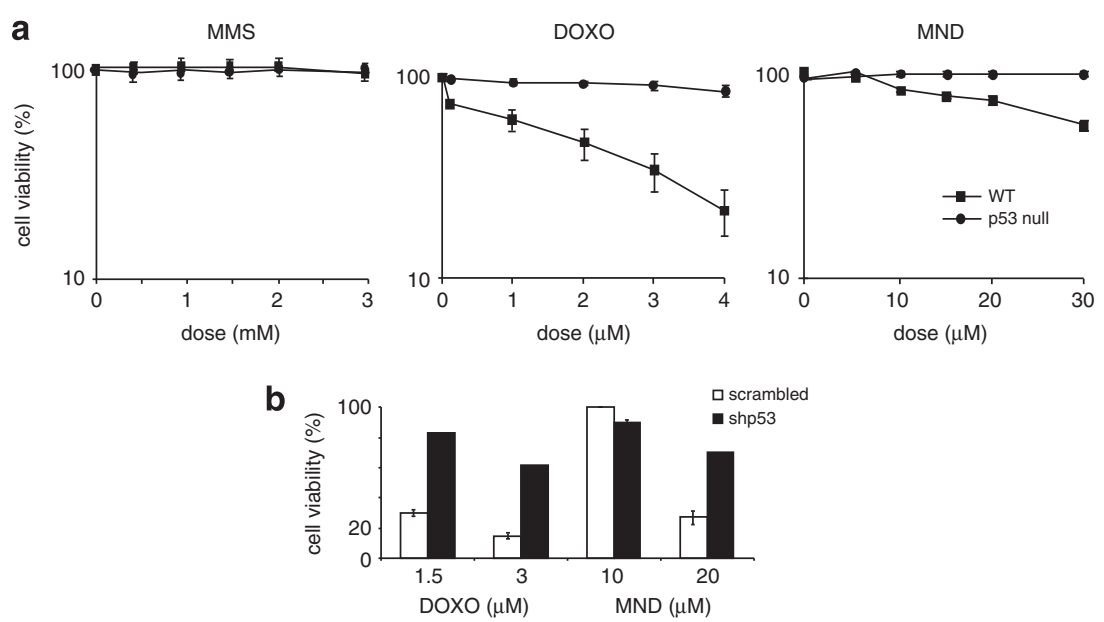

c

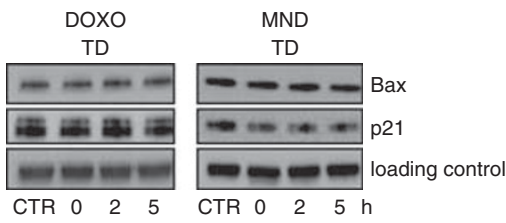

Figure 8 p53 is required for the execution of cell death in myotubes, but the repertoire of genes activated is restricted. (a) Wild-type and p53-null myotubes were exposed to various doses of MMS, DOXO or MND, and cell survival was measured by counting the metabolically active cells by the CCK-8 assay $48 \mathrm{~h}$ after treatment. Error bars indicate S.D. (b) Stable silenced p53 myotubes (transfected with a vector containing a p53 shRNA) and control myotubes (transfected with a vector containing a scrambled sequence) were exposed to DOXO and MND at the indicated doses, and cell survival was measured by counting the metabolically active cells $48 \mathrm{~h}$ after treatment. Error bars indicate S.D. (c) Western blot analysis of Bax ( $23 \mathrm{kDa})$ and p21 (21 kDa) in whole extracts from myotubes exposed to DOXO $(3 \mu \mathrm{M}$ for $3 \mathrm{~h})$ or MND ( $30 \mu \mathrm{M} \mathrm{MND}$ for $1 \mathrm{~h})$. CTR, untreated control cells. Loading control, GAPDH $(37 \mathrm{kDa})$. The estimated MW is indicated for each protein

death by defective signaling downstream of $p 53$, but this blockade is overcome when specific types of damage are induced.

p53 activation is required for the execution of cell death in myotubes. Although p53 is not the unique regulator and executor of the DDR, loss of p53 still compromises the execution of cell fate decisions. This is also the case in post-mitotic muscle cells. When satellite cells were derived from $p 53$ null mice, allowed to differentiate, and then exposed to MMS, DOXO or MND protection from cell killing was recorded after exposure to DOXO and MND, whereas no effect was observed after MMS treatment (Figure 8a). Muscle cells lacking p53 have a reduced ability to differentiate, ${ }^{28}$ however, under our experimental conditions, we were able to achieve a differentiation efficiency above $80 \%$, thus limiting the possible effects of contamination by proliferating cells. To confirm these results, the same experiment was conducted by silencing p53 in satellite cells by short hairpin RNA (shRNA; Figure $8 \mathrm{~b}$ ). A clear protection $(P<0.05)$ from cytotoxicity was observed at the doses of DOXO tested and after $20 \mu \mathrm{M}$ MND. The sensitivity to these agents was higher in the control cells transfected with a scrambled sequence (Figure $8 \mathrm{~b}$ ) as compared with wild-type myotubes (Figure 8a). Interestingly, although the activation of cell death by DOXO and MND is fully p53-dependent, terminally differentiated cells have restricted the repertoire of genes activated by $\mathrm{p} 53$. As shown in Figure 8c, the p53 target genes $p 21$ and (BCL2-associated $X$ protein) Bax are not transactivated following exposure to either DOXO or
MND, whereas transactivation of $p 21$ is observed upon damage in myoblasts (data not shown).

These data confirm that a p53-dependent cell death program is triggered in myotubes upon DOXO- and MND-induced damage, but this pathway is not activated by the SSB-inducing agent.

\section{Discussion}

Post-mitotic cells accumulate DNA damage because of the downregulation of the major DNA repair mechanisms, but are resistant to various stressors. ${ }^{2}$ Our study unveils some of the mechanisms underlying this phenomenon in terminally differentiated muscle cells. We show that ATM-mediated DDR is activated in myotubes by exposure to a variety of DNA-damaging agents, including SSB inducers, but this is not sufficient to trigger cell death in post-mitotic cells. Specific types of damage, such as DSB in transcriptionally active genes or induction of mitochondrial ROS (as induced by DOXO and MND, respectively), are required to activate p53 and the cell death pathway in these cells.

In 2004, Latella et al. $^{14}$ described the activation of ATM-Chk2 pathway in myotubes upon IR exposure. It is largely accepted that DSB either induced directly, or resulting from unrepaired SSB, or the replication-fork collapse initiate the DDR by activating the ATM kinase and DNA-PK, and subsequently, activation of the checkpoint kinase CHK2. Conversely, ssDNA repair intermediates and ssDNA that arises during replication stress recruit the ATR complex, ${ }^{29}$ which subsequently entails the activation of another 
checkpoint kinase $\mathrm{CHK} 1$. The ATR-CHK1 branch is absent in myotubes, but we show that ATM activation and signaling by SSB occurs also in this ATR-defective background (typical of post-mitotic cells). ATM-Chk2 was previously shown to be activated in response to oxidative and alkylation damage in G1 cells. ${ }^{11}$ We provide the first evidence that this occurs also in post-mitotic cells, thus definitively precluding the involvement of DNA replication in the activation of ATM. What are the lesions/events that activate ATM can be only a matter of speculation. The lack of involvement of ATM in the case of early appearing $\gamma \mathrm{H} 2 \mathrm{AX}$ foci induced by MMS suggests that AP sites arising by DNA glycosylase-mediated base removal are unable to activate ATM. A more likely scenario is that these foci represent regions of altered chromatin structure due to BER events. ${ }^{30}$ Conversely, ATM seems to be involved in the appearance of the late $\gamma \mathrm{H} 2 \mathrm{AX}$ foci. We may speculate that the lesions that are responsible for triggering the signaling cascade are produced by the processing of $\mathrm{O}^{6}$-alkylG/C mispairs. In proliferating cells, ATM/ATR activation by methylation damage requires a functional mismatch repair $(\mathrm{MMR}),{ }^{30}$ but what happens in post-mitotic cells that are deprived of $\mathrm{MMR}^{2}$ is an open question. The appearance of $\mathrm{H}_{2} \mathrm{O}_{2}$-induced foci in myotubes is fully dependent on ATM, suggesting that $3^{\prime}$-DNA end-blocking groups, such as $3^{\prime}$-abasic sites and 3'-phosphoglycolates, are efficient activators of the signaling cascade. Interestingly, these lesions are repaired by tyrosyl-DNA phosphodiesterase 1, a well conserved tyrosyl DNA phosphodiesterase that has been shown to be part of the DDR triggered by accumulation of oxidative damage in quiescent fission yeast. ${ }^{31}$ The lack of participation of DNA-PK to the dephosphorylation of $\mathrm{H} 2 \mathrm{AX}$ upon $\mathrm{H}_{2} \mathrm{O}_{2}$-induced damage indicates that $\mathrm{NHEJ}$ is not involved in their repair. Also, CPT activates the $\gamma \mathrm{H} 2 \mathrm{AX}$ response in our post-mitotic muscle cell system (data not shown). CPT is able to trap selectively TOP1cc, which are TOP1-linked DNA SSBs. ${ }^{16}$ Recently, CPT has been shown to induce ATM activation and phosphorylation of the histone $\mathrm{H} 2 \mathrm{AX}$ in post-mitotic neurons and lymphocytes. ${ }^{12} \mathrm{~A}$ mechanism for DDR activation by CPT has been proposed where TOP1cc would produce transcription arrests with R-loop formation and generation of DSBs that would in turn activate ATM and trigger phosphorylation of $\mathrm{H} 2 \mathrm{AX} .^{32}$ Whether this occurs by the same mechanism in muscle cells has not been explored, but the lack of p53 activation indicates that the signaling cascade differs from that triggered by TOP2-mediated DSB (as after DOXO treatment). The selective channeling of ATM-mediated effects towards $\gamma \mathrm{H} 2 \mathrm{AX}$ activation may promote lesion processing and repair, thus contributing to avoid tissue loss. An example is provided by the phosphorylation of XRCC1 by ATM-Chk2 that facilitates recruitment of BER proteins at SSBs. ${ }^{11}$ Interestingly, similarly to what observed after $\mathrm{H}_{2} \mathrm{O}_{2}$ in myotubes, DNA-PK inhibition did not prevent the reversal of $\mathrm{H} 2 \mathrm{AX}$ phosphorylation in lymphocytes exposed to CPT, ${ }^{12}$ suggesting that NHEJ is not involved in the repair of these lesions. Future research should address what are the pathways involved in SSB repair in postmitotic cells.

It has been previously shown ${ }^{14}$ that the radioresistance in myotubes is associated with a blockade of DNA-damage signaling downstream of ATM. Here, we show that the protection by SSB-induced cell death is also associated with defective signaling downstream of ATM. Upon SSB induction, p53 is not activated and its stabilization by nutlin-3 is not sufficient to trigger cell death. Similar to myotubes, neurons are also resistant to a variety of stressors. The so-called restricted apoptosis of mature neurons has been ascribed to the reduction of the levels of Apaf- 1 and to the associated increase in the protective activities of the inhibitors of the apoptosis proteins (IAPs) that sustain survival. ${ }^{33}$ DNA damage has been shown to overcome the inhibition by IAPs via a p53-mediated induction of Apaf-1. ${ }^{34}$ A similar mechanism has been hypothesized for skeletal muscle cells. ${ }^{20}$ However, our data indicate that at least in the case of DNAbreak-inducing stressors, the stabilization of p53 to a degree comparable to genotoxic p53 activation and the associated upregulation of Apaf-1 (by nutlin-3) are not sufficient to trigger cell death. The type of damage and the involved cellular targets seem to have a key role in the activation of this mechanism. Post-mitotic muscle cells activate p53 upon exposure to the TOP2 $\beta$ inhibitor DOXO and to the mitochondria-specific inhibitor MND, and die. p53 is stabilized and phosphorylated on serine 15 (activation of ATM leads to direct phosphorylation of p53 on serine $15^{35}$ ) upon DOXO or MND treatment. It is of note the stabilization of p53 upon MND exposure is in line with the activation of p53 by ROS generated by mitochondria. ${ }^{36}$ This does not occur upon acute treatment of myotubes with $\mathrm{H}_{2} \mathrm{O}_{2}$, suggesting that the functionality of mitochondria (and likely the activation of mitochondrial p53) have a key role in muscle integrity. The use of a ROS scavenger, such as NAC, fully protected myotubes from the toxic effects of MND, indicating that oxidant stress is involved in this response. What type of damage triggers cell death upon DOXO exposure is less defined. Currently, the free radical hypothesis is most favored because of the redox cycling ability of DOXO to generate highly reactive-oxygenfree radicals. ${ }^{37}$ However, the lack of modulation of DOXO toxicity by NAC supports a model in which the proteosomal processing of DOXO-induced TOP $2 \beta$-DNA cc exposes DSB that, if not repaired, are the major contributors to cell death. Similar data have been obtained in cardiomyocytes $^{26}$ in which DOXO-induced DNA damage was shown to involve the TOP2 $\beta$ isozyme that is the only TOP2 isoform expressed in adult heart as well as in our myotubes (data not shown).

Even if p53 is stabilized by DOXO and MND, its function as transcription factor is not fully operational in myotubes as shown by the lack of $p 21$ and Bax transactivation. Similarly, absent or modest activation of p53 target genes such as p21, MDM2 and Bax was reported in $\mathrm{C} 2 \mathrm{C} 12$ myotubes exposed to DOXO. ${ }^{14}$ These findings suggest that the transcriptionindependent mitochondrial p53 program contributes to muscle cell death. ${ }^{38}$ DNA-damage-activated cell death may occur via different pathways, including apoptosis, autophagy and necrosis. Autophagy may have a cell-protective function as during differentiation, but it can also cause cell death when it becomes overactivated or inhibited. The contribution of these different pathways to DNA-damage-induced cell death in post-mitotic cells should be addressed by future studies.

From a broader perspective, our study indicate that the use of antioxidants is unlikely to prevent the cardiotoxicity of 
DOXO. Indeed the effect of oxidative stress in clinical cardiotoxicity is increasingly questioned. ${ }^{39}$ Our study identifies the activation of $\mathrm{p} 53$ by TOP $2 \beta$-linked DSB as the key event for DOXO-induced muscle cell death. Moreover, the identification of the extraordinary resistance of muscle cells to acute treatments with a variety of DNA SSB-inducing agents may help in the design of anti-cancer drugs effective for proliferating cells, but deprived of toxicity, for post-mitotic cells.

\section{Materials and Methods}

Cell growth conditions and treatment. Murine skeletal muscle satellite cells (MSCs) were isolated, cultured and differentiated as described in Tiainen et al. ${ }^{15}$ Briefly, skeletal MSCs were isolated from the hind limb muscles of young FVB;129 mice. MSCs were maintained as actively proliferating adult stem cells (myoblasts) and induced to differentiate in post-mitotic myotubes by modifying the cell growth conditions. In particular, myoblasts were subcultured in the growth medium (GM; F-10 nutrient mixture plus glutamax supplemented with $10 \%$ of fetal calf serum, $100 \mathrm{U} / \mathrm{ml}$ penicillin/streptomycin, $3 \%$ chicken embryo extract (obtained from 10- to 11-day-old embryos) and $2.5 \mu \mathrm{g} / \mathrm{ml}$ of recombinant human FGF-basic (Peprotech, Rocky Hill, NJ, USA)). To induce terminal differentiation, the GM was replaced with the differentiation medium (Dulbecco's modified Eagle medium plus glutamax, supplemented with $10 \%$ fetal calf serum and $100 \mathrm{U} / \mathrm{ml}$ penicillin/ streptomycin). Cells were incubated at $38{ }^{\circ} \mathrm{C}, 10 \% \mathrm{CO}_{2}$. The efficiency of cell differentiation was routinely controlled by staining with MHC antibodies, and evaluation of the differentiation and fusion indexes. The differentiation index is calculated as the percentage of nuclei belonging to MHC-positive cells on total nuclei. The fusion index is calculated as the percentage of nuclei belonging to MHC-positive cells possessing three or more nuclei on total MHC-positive cells.

p53-null myoblasts were a generous gift of Silvia Soddu (Regina Elena Cancer Institute, Rome, Italy). Myoblasts and myotubes were treated with various doses of the DNA-damaging agent at $37^{\circ} \mathrm{C}$ (as indicated in the legends to figures) and harvested at different post-treatment times.

Stable knockdown of p53 in MSC cell line was obtained by transfection of p-retroSuper vector containing a specific shRNA for murine p53 or a scrambled sequence (control cells), kindly provided by Dr Cinzia Rinaldo (Regina Elena Cancer Institute).

p53-shRNA sequence: 5'-gatccccGTACATGTGTAATAGCTCCttcaagagaGGAG CTATTACACATGTACttttggaaa- $3^{\prime}$.

Scrambled sequence: $5^{\prime}$-gatccccCTATAACGCTCGATATttcaagagaATATCGAG CGCCGTTATAGttttggaaa-3'.

Hairpin-forming sequences are in capital letters. Transfection of MSC was performed using Lipofectamine Plus reagent (Invitrogen, Paisley, UK) according to manufacture's instructions; $48 \mathrm{~h}$ post transfection, cells were split $1: 3$ and kept in GM supplemented with $0.1 \mathrm{mg} / \mathrm{ml}$ puromycin (Sigma-Aldrich, Saint Louis, MO, USA) for 1 week.

The ATM inhibitor Ku55933 (Calbiochem, Darmstadt, Germany) and the DNA-PK inhibitor NU7441 (Axon Medchem BV, Groningen, The Netherlands) were used at 10 and $5 \mu \mathrm{M}$ final concentration, respectively. The cells were pre-incubated with the kinase inhibitors in complete medium $1 \mathrm{~h}$ before exposure to the DNA-damaging agent. The p53 stabilizer nutlin-3 (Santa Cruz Biotechnology, Inc., Santa Cruz, CA, USA) was applied at $1 \mu \mathrm{M}$ dose $24 \mathrm{~h}$ after induction of differentiation. Where indicated, the proteasome inhibitor MG132 $(0.5 \mu \mathrm{M})$ and the anti-oxidant NAC $(0.5 \mathrm{mM})$ were added $30 \mathrm{~min}$ before treatment. Both compounds were purchased from Calbiochem.

Analysis of cell viability and DNA damage. Cell viability was determined by counting metabolically active cells by using the kit-8 (CCK-8) purchased by Doijndo Laboratories (Kumamoto, Japan). Briefly, cells were seeded in a 48-well plate and pre-incubated overnight. The following day, cells were treated with the specific chemical and incubated for $48 \mathrm{~h}$ at $38^{\circ} \mathrm{C}, 10 \% \mathrm{CO}_{2}$. CCK8 was added according to technical instructions, and the absorbance at $450 \mathrm{~nm}$ was measured by a microplate reader (Bio-Rad, Segrate, Milano, Italy).

DNA breaks were measured by the comet assay as previously described, ${ }^{40}$ with minor modifications. The immunofluorescence analysis of $\gamma \mathrm{H} 2 \mathrm{AX}$ foci was performed by incubating the cells with mouse monoclonal anti- $\gamma \mathrm{H} 2 \mathrm{AX}$ antibody (Upstate Biotechnology, Lake Placid, NY, USA). Cells were counterstained with Hoechst 33258 dye. Only nuclei showing 10 bright foci were considered positive.
Western blot analysis. Proteins were separated on $4-12 \%$ polyacrylamide gels and analyzed by western blotting with the following antibodies: mouse monoclonal anti-Chk1 (G-4; Santa Cruz Biotechnology, Inc.), rabbit monoclonal anti- $\gamma \mathrm{H} 2 \mathrm{AX}$ (Ser 139; Millipore, Billerica, MA, USA); rabbit polyclonal anti-phospho-p53 Ser15 (human)/18 (mouse) antibody (Millipore); rabbit polyclonal anti-p53 antibody (a gift of PG Pelicci, European Institute of Oncology, Milan, Italy), mouse monoclonal anti-p21 (F-5; Santa Cruz Biotechnology, Inc.), rat monoclonal anti-APAF-1 (Chemicon International Inc., Temecula, CA, USA), rabbit polyclonal anti-Bax (Santa Cruz Biotechnology, Inc.), mouse monoclonal anti-myogenin (F5D; Santa Cruz Biotechnology, Inc.), mouse monoclonal antiGAPDH (6C5; Santa Cruz Biotechnology, Inc.) and rabbit polyclonal anti-HAP (C-20; Santa Cruz Biotechnology, Inc.). Western blots were developed by using the West Dura kit (Pierce Chemical, Rockford, IL, USA).

\section{Conflict of Interest}

The authors declare no conflict of interest.

Acknowledgements. This work was supported by Associazione Italiana per la Ricerca sul Cancro, Progetto Integrato Oncologia (Ministry of Public Health) and Programma ISS-NIH.

1. Ciccia A, Elledge SJ. The DNA damage response: making it safe to play with knives. Mol Cell 2010; 40: 179-204.

2. Fortini $P$, Dogliotti $E$. Mechanisms of dealing with DNA damage in terminally differentiated cells. Mutat Res 2010; 685: 38-44.

3. Narciso L, Fortini P, Pajalunga D, Franchitto A, Liu P, Degan $P$ et al. Terminally differentiated muscle cells are defective in base excision DNA repair and hypersensitive to oxygen injury. Proc Natl Acad Sci USA 2007; 104: 17010-17015.

4. Jin R, Sun Y, Qi X, Zhang H, Zhang Y, Li N et al. E2F1 is involved in DNA single-strand break repair through cell-cycle-dependent upregulation of XRCC1 expression. DNA Repair 2011; 10: 926-933.

5. Hardeland U, Kunz C, Focke F, Szadkowski M, Schär P. Cell cycle regulation as a mechanism for functional separation of the apparently redundant uracil DNA glycosylases TDG and UNG2. Nucleic Acids Res 2007; 35: 3859-3867.

6. Nouspikel T, Hanawalt PC. Impaired nucleotide excision repair upon macrophage differentiation is corrected by E1 ubiquitin-activating enzyme. Proc Natl Acad Sci USA 2006; 103: 16188-16193.

7. Smith J, Tho LM, Xu N, Gillespie DA. The ATM-Chk2 and ATR-Chk1 pathways in DNA damage signaling and cancer. Adv Cancer Res 2010; 108: 73-112.

8. Bonner WM, Redon CE, Dickey JS, Nakamura AJ, Sedelnikova OA, Solier S, Pommier Y. $\gamma \mathrm{H} 2 \mathrm{AX}$ and cancer. Nat Rev Cancer 2008; 8: 957-967.

9. Caldecott KW. Single-strand break repair and genetic disease. Nat Rev Genet 2008; 9: 619-631.

10. Sjakste N, Sjakste T. Possible involvement of DNA strand breaks in regulation of cell differentiation. Eur J of Histochem 2007; 51: 81-94.

11. Chou WC, Wang HC, Wong FH, Ding SL, Wu PE, Shieh SY et al. Chk2-dependent phosphorylation of XRCC1 in the DNA damage response promotes base excision repair. EMBO J 2008; 27: 3140-3150.

12. Sordet O, Redon CE, Guirouilh-Barbat J, Smith S, Solier S, Douarre C et al. Ataxia telangiectasia mutated activation by transcription- and topoisomerase l-induced DNA double-strand breaks. EMBO Rep 2009; 10: 887-893.

13. Tian $B$, Yang $Q$, Mao Z. Phosphorylation of ATM by Cdk5 mediates DNA damage signalling and regulates neuronal death. Nat Cell Biol 2009; 11: 211-218.

14. Latella L, Lukas J, Simone C, Puri PL, Bartek J. Differentiation-induced radioresistance in muscle cells. Mol Cell Biol 2004; 24: 6350-6361.

15. Tiainen M, Pajalunga D, Ferrantelli F, Soddu S, Salvatori G, Sacchi A et al. Terminally differentiated skeletal myotubes are not confined to G0 but can enter G1 upon growth factor stimulation. Cell Growth Differ 1996; 7: 1039-1050.

16. Pommier $\mathrm{Y}$, Leo $\mathrm{E}$, Zhang $\mathrm{H}$, Marchand $\mathrm{C}$. DNA topoisomerases and their poisoning by anticancer and antibacterial drugs. Chem Biol 2010; 17: 421-433.

17. Kuster GM, Häuselmann SP, Rosc-Schlüter BI, Lorenz V, Pfister O. Reactive oxygen/ nitrogen species and the myocardial cell homeostasis: an ambiguous relationship. Antioxid Redox Signal 2010; 13: 1899-1910.

18. Fisher CL, Fisher AG. Chromatin states in pluripotent, differentiated, and reprogrammed cells. Curr Opin Genet Dev 2011; 21: 140-146.

19. Pajalunga D, Mazzola A, Salzano AM, Biferi MG, De Luca G, Crescenzi M. Critical requirement for cell cycle inhibitors in sustaining nonproliferative states. J Cell Biol 2007; 176: 807-818.

20. Smith MI, Huang YY, Deshmukh M. Skeletal muscle differentiation evokes endogenous XIAP to restrict the apoptotic pathway. PLOS One 2009; 4: e5097. 
21. Lukas C, Bartkova J, Latella L, Falck J, Mailand N, Schroeder T et al. DNA damageactivated kinase Chk2 is independent of proliferation or differentiation yet correlates with tissue biology. Cancer Res 2001; 61: 4990-4993.

22. Puri PL, Sartorelli V. Regulation of muscle regulatory factors by DNA-binding, interacting proteins, and post-transcriptional modifications. J Cell Physiol 2000; 185: 155-173.

23. Caspari T. How to activate p53. Curr Biol 2000; 10: R315-R317.

24. Shangary S, Wang S. Small-molecule inhibitors of the MDM2-p53 protein-protein interaction to reactivate p53 function: a novel approach for cancer therapy. Annu Rev Pharmacol Toxicol 2009; 49: 223-241.

25. Zhang A, Lyu YL, Lin CP, Zhou N, Azarova AM, Wood LM et al. A protease pathway for the repair of topoisomerase II-DNA covalent complexes. J Biol Chem 2006; 281: 35997-36003.

26. Lyu YL, Kerrigan JE, Lin CP, Azarova AM, Tsai YC, Ban Y et al. Topoisomerase Ilbeta mediated DNA double-strand breaks: implications in doxorubicin cardiotoxicity an prevention by dexrazoxane. Cancer Res 2007; 67: 8839-8846.

27. Loor G, Kondapalli J, Schriewer JM, Chandel NS, Vanden Hoek TL, Schumacker PT. Menadione triggers cell death through ROS-dependent mechanisms involving PARP activation without requiring apoptosis. Free Radic Biol Med 2010; 49: 1925-1936.

28. Soddu S, Blandino G, Scardigli R, Coen S, Marchetti A, Rizzo MG et al. Interference with p53 protein inhibits hematopoietic and muscle differentiation. J Cell Biol 1996; 134: 193-204

29. López-Contreras AJ, Fernandez-Capetillo O. The ATR barrier to replication-born DNA damage. DNA Repair (Amst) 2010; 9: 1249-1255.

30. Stojic L, Mojas N, Cejka P, di Pietro M, Ferrari S, Marra G et al. Mismatch-repair-dependen G2 checkpoint induced by low doses of SN1 type methylating agents requires the ATR kinase. Genes Dev 2004; 18: 1331-1344.
31. Arcangioli $B$, Ben Hassine S. Unrepaired oxidative DNA damage induces an ATR/ATM apoptotic-like response in quiescent fission yeast. Cell Cycle 2009; 8: 2326-2331.

32. Sordet O, Nakamura AJ, Redon CE, Pommier Y. DNA double-strand breaks and ATM activation by transcription-blocking DNA lesions. Cell Cycle 2010; 9: 274-278.

33. Lindholm D, Arumäe U. Cell differentiation: reciprocal regulation of Apaf-1 and the inhibitor of apoptosis proteins. J Cell Biol 2004; 167: 193-195.

34. Vaughn $A E$, Deshmukh $M$. Essential postmitochondrial function of p53 uncovered in DNA damage-induced apoptosis in neurons. Cell Death Differ 2007; 14: 973-981.

35. Siliciano JD, Canman CE, Taya Y, Sakaguchi K, Appella E, Kastan MB. DNA damage induces phosphorylation of the amino terminus of p53. Genes Dev 1997; 11: 3471-3481.

36. Nithipongvanitch R, Ittarat W, Cole MP, Tangpong J, Clair DK, Oberley TD. Mitochondrial and nuclear p53 localization in cardiomyocytes: redox modulation by doxorubicin (Adriamycin)? Antioxid Redox Signal 2007; 9: 1001-1008.

37. Simůnek T, Stérba M, Popelová O, Adamcová M, Hrdina R, GersIV. Anthracycline-induced cardiotoxicity: overview of studies examining the roles of oxidative stress and free cellular iron. Pharmacol Rep 2009; 61: 154-171.

38. Vaseva AV, Moll UM. The mitochondrial p53 pathway. Biochim Biophys Acta 2009; 1787: 414-420.

39. Zhang Y-W, Shi J, Li Y-J, Wei L. Cardiomyocyte death in doxorubicin-induced cardiotoxicity. Arch Immunol Ther Exp 2009; 57: 435-445.

40. Fortini $P$, Pascucci B, Belisario F, Dogliotti E. DNA polymerase beta is required for efficient DNA strand break repair induced by methyl methanesulfonate but not by hydrogen peroxide. Nucl Acids Res 2000; 28: 3040-3046

Supplementary Information accompanies the paper on Cell Death and Differentiation website (http://www.nature.com/cdd) 